\section{Defects that Control the Properties of Tl- and Hg-based Superconductors}

J. D. Jorgensen ${ }^{a}$, O. Chmaissem ${ }^{b}$, J. L. Wagnerb,c, W. R. Jensen ${ }^{c}$, B. Dabrowskia,d, D. G. Hinks ${ }^{\text {a }}$, and J. F. Mitchell ${ }^{\mathrm{a}}$

aMaterials Science Division

Argonne National Laboratory, Argonne, IL 60439

bScience and Technology Center for Superconductivity

Argonne National Laboratory, Argonne, IL 60439

cDepartment of Physics

University of North Dakota, P. O. Box 7129, Grand Forks, ND 58202

dPhysics Department

Northem Illinois University, DeKalb, IL 60115

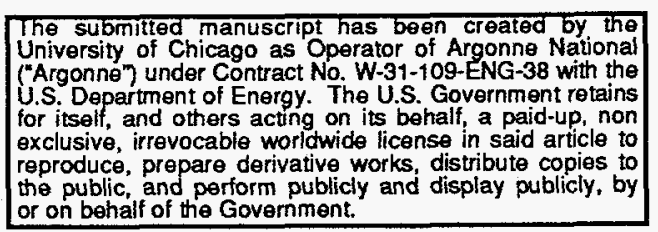

March, 1997

To be published in Physica $\mathrm{C}$

$/ \mathrm{sm}$

\author{
DISCLAIMER
}

\section{Distribution:}

$\begin{aligned} \text { 1-3. } & \text { M. J. Masek } \\ \text { 4. } & \text { B. D. Dunlap } \\ \text { 5. } & \text { G. W. Crabtree } \\ \text { 6. } & \text { A. A. Abrikosov } \\ \text { 7. } & \text { F. Y. Fradin } \\ \text { 8. } & \text { M. B. Brodsky } \\ \text { 9. } & \text { Editorial Office } \\ \text { 10. } & \text { Authors } \\ \text { 11. } & \text { S\&TC (5) } \\ \text { 12. } & \text { High Tc }\end{aligned}$

\begin{abstract}
This report was prepared as an account of work sponsored by an agency of the United States Government. Neither the United States Government nor any agency thereof, nor any of their employees, makes any warranty, express or implied, or assumes any legal liability or responsibility for the accuracy, completeness, or usefulness of any information, apparatus, product, or process disclosed, or represents that its use would not infringe privately owned rights. Reference herein to any specific commercial product, process, or service by trade name, trademark, manufacturer, or otherwise does not necessarily constitute or imply its endorsement, recommendation, or favoring by the United States Government or any agency thereof. The views and opinions of authors expressed herein do not necessarily state or reflect those of the United States Government or any agency thereof.
\end{abstract}

INVITED PAPER submitted to the 5th International Conference on Materials and Mechanisms of Superconductivity, High-Temperature Superconductors, Beijing, China, February 28-March 4, 1997

This work is supported by the Division of Materials Sciences, Office of Basic Energy Sciences of DOE, under contract No. W-31-109-ENG-38 (JDJ, BD, DGH, JFM) and the National Science Foundation, Office of Science and Technology Centers, under grant \#DMR 91-20000 (OC). 
Defects that Control the Properties of Tl- and Hg-based Superconductors

J. D. Jorgensen ${ }^{a}$, O. Chmaissem ${ }^{b}$, J. L. Wagner bc, W. R. Jensen c, B. Dabrowskia,d, D. G. Kinks ${ }^{a}$, and J. F. Mitchell

a Materials Science Division, Argonne National Laboratory, Argonne, IL 60439

b Science and Technology Center for Superconductivity, Argonne National Laboratory, Argonne, II 60439

${ }^{\mathrm{C} D e p a r t m e n t ~ o f ~ P h y s i c s, ~ U n i v e r s i t y ~ o f ~ N o r t h ~ D a k o t a, ~ P . ~ O . ~ B o x ~ 7129, ~ G r a n d ~ F o r k s, ~ N D ~} 58202$ dPhysics Department, Norther Illinois University, DeKalb, II 60115.

Invited paper submitted to the Fth International Conference on Materials and Mechanisms of Superconductivity - High-Temperature

Superconductors, Beijing, China, February 28 - March 4, 1997; to be published in Physical C. 


\section{Defects that Control the Properties of Tl- and Hg-based Superconductors}

J. D. Jorgensen ${ }^{a}$, O. Chmaissem ${ }^{b}$, J. L. Wagner ${ }^{b, c}$, W. R. Jensenc, B. Dabrowskia,d, D. G. Hinks ${ }^{a}$, and J. F. Mitchella

aMaterials Science Division, Argonne National Laboratory, Argonne, Il 60439

bScience and Technology Center for Superconductivity, Argonne National Laboratory, Argonne, IL 60439

'Department of Physics, University of North Dakota, P. O. Box 7129, Grand Forks, ND 58202

dPhysics Department, Northern Illinois University, DeKalb, IL 60115.

Defects that affect $\mathrm{T}_{\mathrm{c}}$ in $\mathrm{Tl}_{2} \mathrm{Ba}_{2} \mathrm{CuO}_{6+\delta}$ and $\mathrm{HgBa}_{2} \mathrm{CuO}_{4+\delta}$ have been characterized by neutron powder diffraction. In $\mathrm{Tl}_{2} \mathrm{Ba}_{2} \mathrm{CuO}_{6+\delta}$, the dominant defect is interstitial oxygen between the two $\mathrm{Tl}-\mathrm{O}$ planes, but $\mathrm{Cu}$ substitution on the $\mathrm{Tl}$ site also affects properties and there is evidence for a second oxygen defect for compositions in the vicinity of maximum $\mathrm{T}_{\mathrm{c}}$. In $\mathrm{HgBa}_{2} \mathrm{CuO}_{4+\delta}$, there are two competing oxygen defects in the $\mathrm{Hg}$ layer. The relative concentrations of these defects switches upon passing from the underdoped region, through the maximum $T_{c}$, to the overdoped region. This remarkable behavior could result from a change in the topology of the Fermi surface upon passing through the van Hove singularity.

\section{INTRODUCTION}

The $\mathrm{Tl}_{2} \mathrm{Ba}_{2} \mathrm{CuO}_{6+\delta} \quad(\mathrm{Tl}-2201) \quad[1]$ and $\mathrm{HgBa}_{2} \mathrm{CuO}_{4+\delta}(\mathrm{Hg}-1201)$ [2] superconducting compounds are of particular importance for the study of the physics of HTS because these materials exhibit the highest $\mathrm{T}_{c}$ 's observed for any material with one $\mathrm{CuO}_{2}$ layer, they have simple crystal structures, and the doping can be varied over a wide range. For Tl2201 , the maximum $T_{c}$ is $\sim 94 \mathrm{~K}$ and the compound can be overdoped into the normal-metal regime. For $\mathrm{Hg}-1201$, the maximum $\mathrm{T}_{\mathrm{c}}$ is $\sim 98 \mathrm{~K}$ and the compound can be both underdoped and overdoped.

To enable the reproducible synthesis of these materials and to interpret the physical measurements, one must understand the defects that control the properties. Multiple defects form in both compounds. In this paper, we describe these defects and their effects on $T_{c}$. We also comment on what this complex defect chemistry may teach us about the underlying electronic structure - specifically, that the competition between slightly different oxygen defects can be strongly affected by crossing the van Hove singularity.

\section{2. $\mathrm{Tl}_{2} \mathrm{Ba}_{2} \mathrm{CuO}_{6+\delta}$}

Two defects have previously been described in the literature for $\mathrm{Tl}-2201$ [3]. $\mathrm{T}_{c}$ is affected most strongly by an interstitial oxygen defect $\mathrm{O} 4$ located between the two Tl-O layers. $T_{c}$ is maximum $(-94 \mathrm{~K})$ for the minimum amount of defect oxygen and decreases as more oxygen is added, eventually reaching the normal metallic state.

The second defect is $\mathrm{Cu}$ substitution at the $\mathrm{Tl}$ site. This defect was discussed in some of the earliest papers about Tl-2201, but the experimental evidence was not strong. More recently, Aranda et al. [4] used anomalous X-ray diffraction (at the $\mathrm{Cu}$ edge) to prove the existence of this defect. By comparing two samples, they showed that the unit cell tends to have a smaller orthorhombic strain (or appear tetragonal within experimental resolution) for higher concentrations of this defect. However, they did not correlate superconducting properties to the concentration of this defect.

To investigate the defect chemistry more fully, we prepared samples of Tl-2201 by a conventional solid-state-reaction technique for various $\mathrm{TV} / \mathrm{Cu}$ starting compositions from 2.3/1.0 to 2.0/1.1 [5]. Portions of each of these samples were then annealed in oxygen at $400^{\circ} \mathrm{C}$ and various oxygen partial pressures to vary the oxygen contents. This synthesis technique yielded samples with a wide range of structural and superconducting properties. For each sample, the amount of oxygen on the $\mathrm{O} 4$ interstitial site was determined by Rietveld refinement using neutron powder diffraction data.
The submitted manuscript has been created by the University of Chicago as Operator of Argonne National Laboratory ("Argonne") under Contract No. W-31-109-ENG-38 with the U.S. Department of Energy. The U.S. Government retains for itself, and others acting on its behalf, a paid-up, nonexclusive, irrevocable worldwide license in said article to reproduce, prepare derivative works, distribute copies to the public, and perform publicly and display publicly, by or on behalf of the Government. 
The $T_{c}$ 's of these samples vs. the oxygen content on the $\mathrm{O} 4$ site are shown in Fig. 1. From the wide scatter in the data, it is immediately obvious that O4 oxygen content is not the only factor controlling $T_{c}$. The $T_{c}$ 's and also the structural data show that these differences result from the different $\mathrm{TV} / \mathrm{Cu}$ starting compositions. We assume, but cannot prove directly from the neutron diffraction data, that these samples differ in the amount of $\mathrm{Cu}$ on the $\mathrm{Tl}$ site. The variation of orthorhombic strains vs. TVCu ratio for our samples (see Fig. 2) agrees with the results of Aranda et al. [4], supporting our assumption. Samples with more $\mathrm{Cu}$ on the $\mathrm{Tl}$ site have smaller orthorhombic strains. For these samples, the maximum $T_{c}$ that can be achieved by varying the oxygen content is also slightly $\left(5-10^{\circ} \mathrm{K}\right)$ lower.

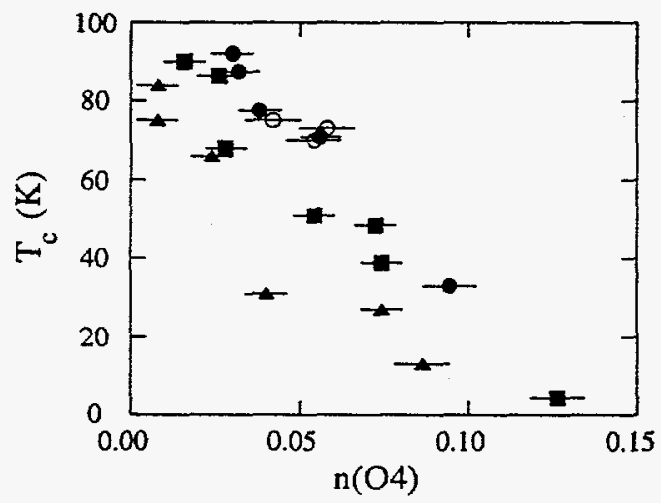

Fig. 1. $\mathrm{T}_{\mathrm{c}}$ vs. oxygen content at the $\mathrm{O} 4$ site for $\mathrm{Tl}-$ 2201. The different symbols are for samples with different $\mathrm{T} / \mathrm{Cu}$ starting compositions.

At oxygen contents near those that give the maximum $T_{c}$, we see evidence for a second oxygen defect. In the vicinity of maximum $T_{c}$, with continuously decreasing oxygen content, the c-axis lattice parameter increases, passes through a maximum, and then decreases again (see Fig. 3). Such behavior is not consistent with a monotonically changing concentration of a single defect. By comparing the oxygen content on the $\mathrm{O} 4$ site (determined by neutron diffraction) with the total oxygen uptake determined by TGA, we conclude that, for these low oxygen concentrations, there is apparently an oxygen defect that is not at the $\mathrm{O} 4$ site. We have been unable to locate this second oxygen defect by neutron diffraction (which is not surprising, due to its small concentration). However, the observation of a similar phenomenon in $\mathrm{Hg}-1201$ at compositions in the vicinity of maximum $T_{c}$ (to be discussed in the next section) suggests that our conclusions are correct and may provide some clues about what different kind of oxygen defect is being formed.

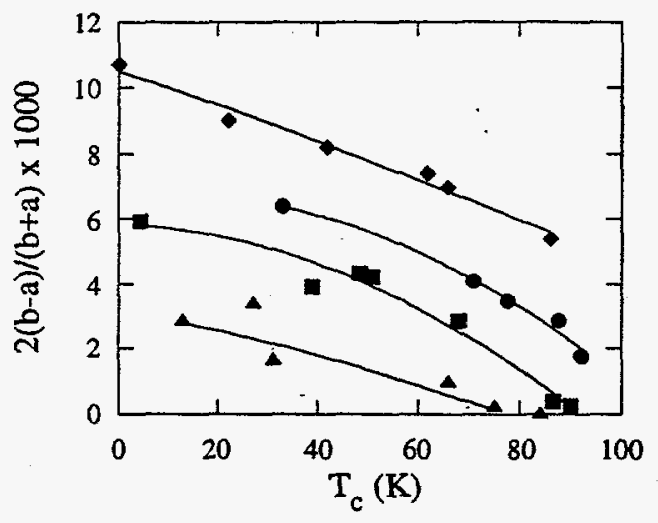

Fig. 2. Orthorhombic strain vs. $T_{c}$ for $T l-2201$ samples with different $\mathrm{Tl} / \mathrm{Cu}$ starting compositions and oxygen contents: closed circles and closed squares, $\mathrm{T} l / \mathrm{Cu}=2.0 / 1.0$, closed triangles $2.0 / 1.1$; diamonds are the data of Ref. 3.

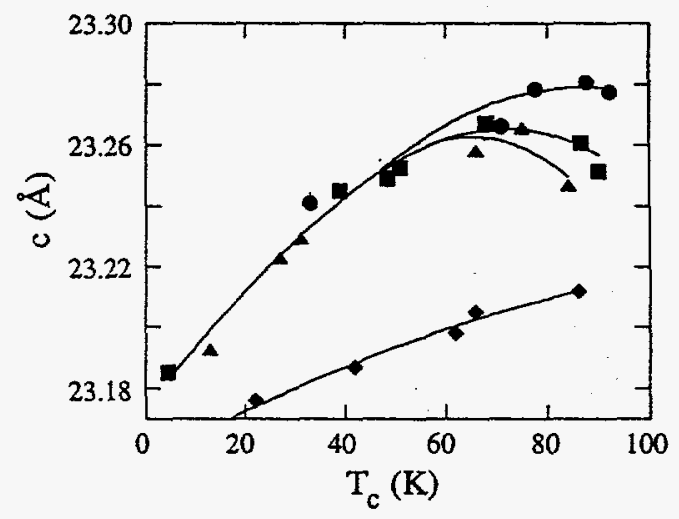

Fig. 3. c-axis length vs. $T_{c}$ for $T 1-2201$ samples (same format as Fig. 2).

\section{3. $\mathrm{HgBa}_{2} \mathrm{CuO}_{4}+\delta$}

Less is known about the defect chemistry of $\mathrm{Hg}$ 1201 than for Tl-2201. There is general agreement that the defect that most influences $T_{c}$ is an interstitial oxygen defect in the center of the $\mathrm{Hg}$ plane. There are conflicting reports that $\mathrm{Cu}$ may substitute at the $\mathrm{Hg}$ site with additional oxygen bonded to this $\mathrm{Cu}$ [6-8]. In this work, we do not investigate this $\mathrm{Hg}$-site substitutional defect. We focus on the oxy- 
gen defect chemistry as the oxygen content is varied over a wide range extending from the underdoped to the overdoped region. We see a remarkable change in the oxygen defect chemistry upon passing through the maximum $T_{c}$.

To avoid confusion from possible differences in metal-site defect chemistry, the measurements reported here were performed by oxygen annealing small samples from a single larger sample. Repeated annealings of the same small samples confirmed that the results are reproducible and do not depend significantly on sample history. The oxygen anneals were performed at $300-400^{\circ} \mathrm{C}$ in oxygen pressures from $10^{-7}$ to $150 \mathrm{~atm}$. Structural parameters were obtained by Rietveld refinement using neutron powder diffraction data obtained on the SEPD at IPNS [9].

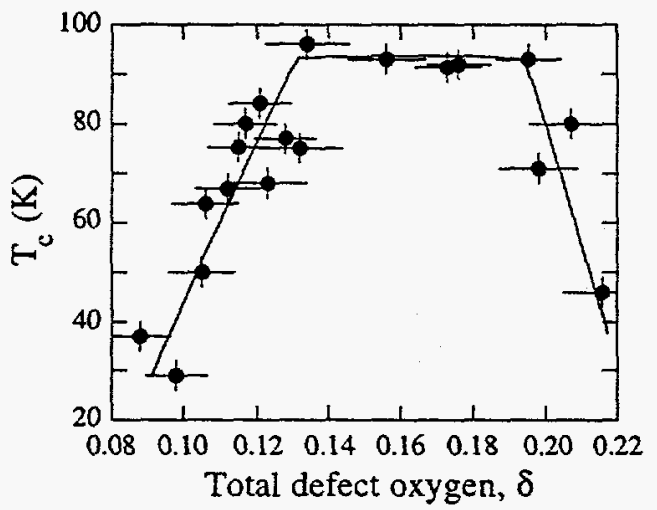

Fig. 4. $T_{c}$ vs, total defect oxygen content, $\delta$, for $\mathrm{Hg}-1201$ samples annealed in various oxygen environments.

Two slightly different oxygen defects were identified in the Rietveld refinements. One of these is the previously reported interstitial oxygen site in the $\mathrm{Hg}$ plane (site $\mathrm{O} 3$ ) $[2,6-8]$. The second site $(\mathrm{O} 4)$ is also in the $\mathrm{Hg}$ plane, but is at an $(x, x, 0)$ site that is displaced towards one of the $\mathrm{Hg}$ atoms, and the $\mathrm{Hg}$ atom is displaced slightly in the same direction, to form a $\mathrm{Hg}-\mathrm{O} 4$ bond $2 \AA$ in length.

The $T_{c}$ vs. total defect oxygen content, $\delta$ (combined occupancies of the $\mathrm{O} 3$ and $\mathrm{O} 4$ sites determined from neutron powder diffraction), is shown in Fig. 4. Data from the present samples are in good agreement with those from the literature, when total defect oxygen contents are used. A surprising feature of the data is the plateau at maximum $T_{c}$, where $T_{c}$ is nominally constant $(>90 \mathrm{~K})$ for total oxygen contents from 0.13 to 0.19 .
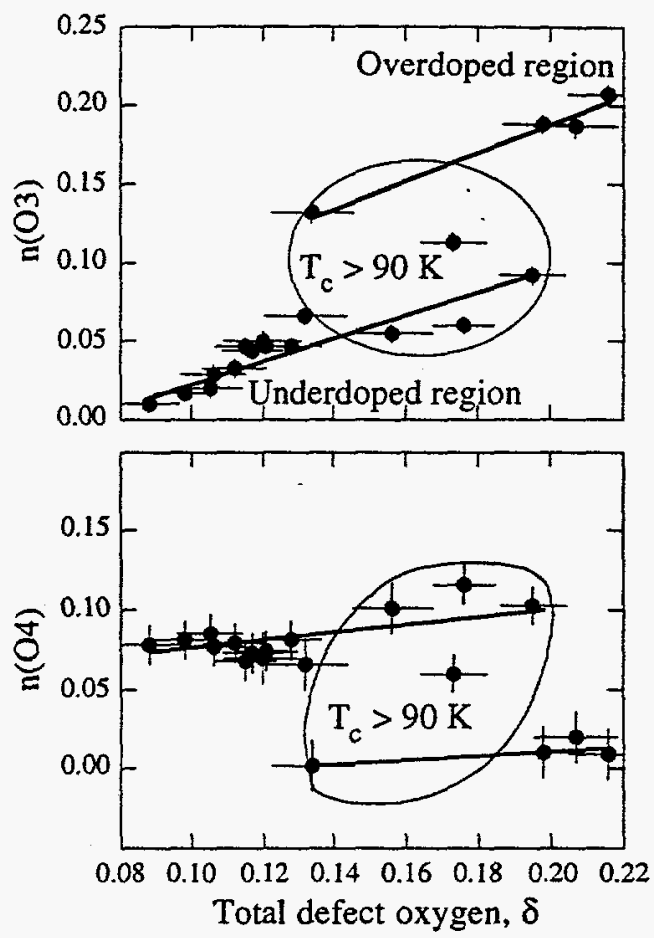

Fig. 5. $\mathrm{O} 3$ and $\mathrm{O} 4$ oxygen defect concentrations vs. total oxygen defect concentration $[\delta=n(O 3)+n(O 4)]$ for $\mathrm{Hg}-1201$.

This plateau results from an unusual competition between the two oxygen defects, illustrated in Fig. 5. For underdoped samples $(\delta<0.13)$, the dominant defect is $\mathrm{O} 4$; for overdoped samples $(\delta>0.19)$, the dominant defect is 03 . This unusual behavior in the defect chemistry also manifests itself in other structural parameters such as cell parameters and positions of the apical oxygen (O2) and $\mathrm{Ba}$ atoms. For example, the unit cell volume vs. $\delta$ is shown in Fig. 6. The monotonic behavior in the underdoped and overdoped regions is separated by a region (corresponding to $\mathrm{T}_{\mathrm{c}}>90 \mathrm{~K}$ ) where the cell volume is nominally constant while the total oxygen content is changing.

From the bond lengths, the charge on $\mathrm{Cu}$ can be estimated by the bond valence sum method. The result of such a calculation is shown in Fig. 7. The charge on $\mathrm{Cu}$ increases with increasing oxygen content in both the underdoped and overdoped regions, but remains nominally constant for the plateau region $(0.13 \leq \delta \leq 0.19)$ where $T_{c}$ is constant. Thus, the 
$T_{\mathfrak{c}}$ is controlled by the number of carriers and the number of carriers remains constant upon passing through the range of oxygen contents where the dominant defect switches from $\mathrm{O} 4$ to $\mathrm{O} 3$.

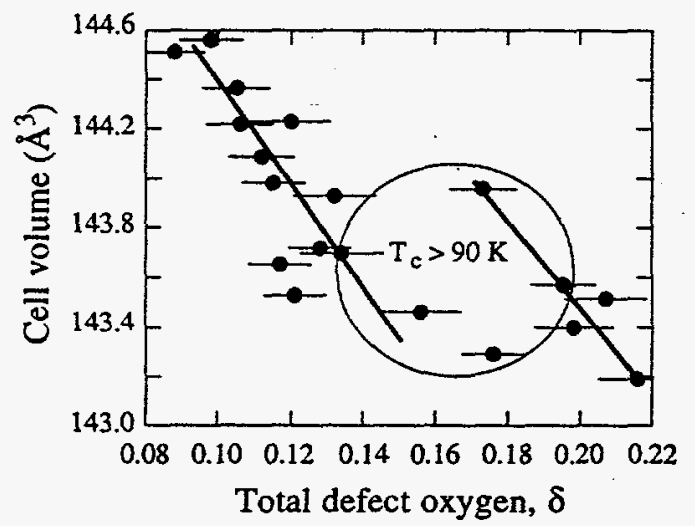

Fig. 6. Unit cell volume vs. total oxygen defect content, $\delta$, for $\mathrm{Hg}-1201$.

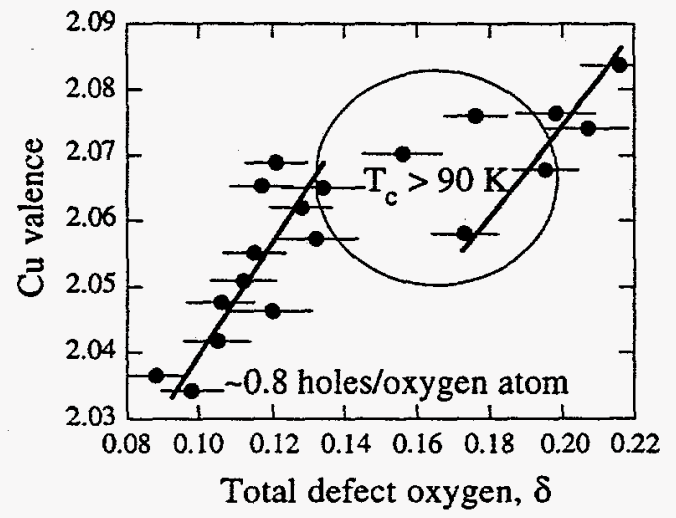

Fig. 7. Bond valence sum for $\mathrm{Cu}$ in $\mathrm{Hg}-1201$ vs. total oxygen defect content, $\delta$.

\section{CONCLUSIONS}

These data suggest that the formation energies for $\mathrm{O} 3$ and $\mathrm{O} 4$ oxygen defects in $\mathrm{Hg}-1201$ differ by only a small amount and that this delicate energy balance is modified upon passing through the region of maximum $T_{c}$. A similar behavior (the appearance of a second oxygen defect in the region of maximum $\mathrm{T}_{\mathrm{c}}$ ) is suggested by our data for the Tl-2201 system, but the data are much less clear in that case because the underdoped region cannot be accessed. Such behavior could result from crossing the van Hove singularity at maximum $T_{\mathrm{c}}$. The resulting topological transition in the Fermi surface could affect the defect chemistry by altering bands involving $\mathrm{Hg}$ (or $\mathrm{Tl}$ ). This conjecture remains to be validated; but, if it is correct, the present data may be the first evidence for crossing the van Hove singularity at maximum $T_{c}$ by varying the chemical composition. Such transitions have not been predicted to result in first-order changes in structural parameters [10]. But, the remarkable behavior observed here could result from the first-order response of the delicately balanced defect chemistry to the changes in electronic structure.

\section{ACKNOWLEDGEMENTS}

We would like to- thank Simine Short for her help with neutron data collection. This work was supported by the US Dept. of Energy, Basic Energy Sciences - Materials Sciences, contract No. W-31109-ENG-38, and the NSF Office of Science and Technology Centers, grant No. DMR 91-20000.

\section{REFERENCES}

1. Z.Z. Sheng, and A.M. Hermann, Nature, 332 (1988) 55.

2. S.N. Putilin, E.V. Antipov, O. Chmaissem, and M. Marezio, Nature, 362 (1993) 226.

3. Y. Shimakawa, Physica C, 204 (1993) 247.

4. M.A.G. Aranda, D.C. Sinclair, J.P. Attfield, and A.P. Mackenzie, Phys. Rev. B, 51 (1995) 12747.

5. J.L. Wagner, O. Chmaissem, J.D. Jorgensen, D.G. Hinks, P.G. Radaelli, B.A. Hunter, and W.R. Jensen, Physica C, in press.

6. J.L. Wagner, P.G. Radaelli, D.G. Hinks, J.D. Jorgensen, J.F. Mitchell, B. Dabrowski, G.S. Knapp, and M. Beno, Physica C, 210 (1993) 447.

7. A. Asab, A.R. Armstrong, I. Gameson, and P.P. Edwards, Physica C, 255 (1995) 180.

8. O. Chmaissem, Q. Huang, A. Santoro, S.N. Putilin, and M. Marezio, Physica C, 212 (1993) 259.

9. J.D. Jorgensen, J. Faber, Jr., J.M. Carpenter, R.K. Crawford, J.R. Haumann, R.L. Hitterman, R. Kleb, G.E. Ostrowski, F.J. Rotella, and T.G. Worlton, J. Appl. Crystallogr., 22 (1989) 321.

10.I.M. Lifshitz, Zh. Eksp. Teor. Fiz., 38 (1960) 1569; [Sov. Phys.-JETP, 11 (1960) 1130]. 


\section{DISCLAIMER}

Portions of this document may be illegible in electronic image products. Images are produced from the best available original docoment. 